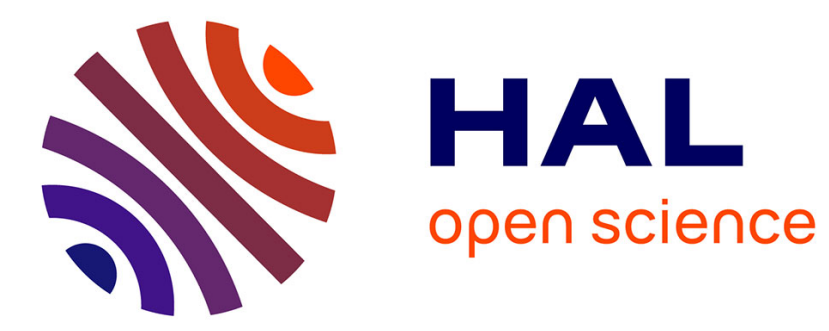

\title{
Two Reliability Acceptance Sampling Plans for Items Subject to Wiener Process of Degradation
}

\author{
Ji Hwan Cha, Sophie Mercier
}

\section{To cite this version:}

Ji Hwan Cha, Sophie Mercier. Two Reliability Acceptance Sampling Plans for Items Subject to Wiener Process of Degradation. Methodology and Computing in Applied Probability, 2022, 24, pp.1651-1668. 10.1007/s11009-021-09879-1 . hal-03268547

\section{HAL Id: hal-03268547 \\ https://hal.science/hal-03268547}

Submitted on 23 Jun 2021

HAL is a multi-disciplinary open access archive for the deposit and dissemination of scientific research documents, whether they are published or not. The documents may come from teaching and research institutions in France or abroad, or from public or private research centers.
L'archive ouverte pluridisciplinaire HAL, est destinée au dépôt et à la diffusion de documents scientifiques de niveau recherche, publiés ou non, émanant des établissements d'enseignement et de recherche français ou étrangers, des laboratoires publics ou privés. 


\title{
Two Reliability Acceptance Sampling Plans for Items Subject to Wiener Process of Degradation
}

\author{
Ji Hwan Cha*1 \\ Department of Statistics, Ewha Womans University \\ Seoul, 120-750, Rep. of Korea \\ e-mail: jhcha@ewha.ac.kr \\ Sophie Mercier \\ Laboratory of Mathematics and their Applications of Pau (LMAP) \\ University of Pau and Pays of Adour / IPRA / CNRS / E2S UPPA \\ 64000 Pau, France \\ e-mail: sophie.mercier@univ-pau.fr
}

\begin{abstract}
Traditionally, in reliability acceptance sampling plans, the decision to accept or reject a lot is made by performing the life tests of items. However, when the item's deterioration is described by a degradation process, it can be made based on the observed deterioration levels of the items obtained from degradation tests. In this paper, two acceptance sampling plans are developed, based on the observation of the deterioration of the items, accumulated on a given period of time. To model the degradation of the items over time, the Wiener process with positive drift is employed. Algorithms to find the parameters of the proposed sampling plans are suggested. Conditionally on the acceptance in the test, the developed sampling plans are shown to improve the reliability performance of the items in the sense that the lifetimes of the items after the reliability sampling test are stochastically larger than those before the test. Also, we compare the two sampling plans both from a technical and economical points of view.
\end{abstract}

Keywords: Quality management; variables sampling plan; degradation test; Wiener process; stochastic ordering

\footnotetext{
${ }^{1}$ Corresponding author
} 


\section{NOTATIONS}

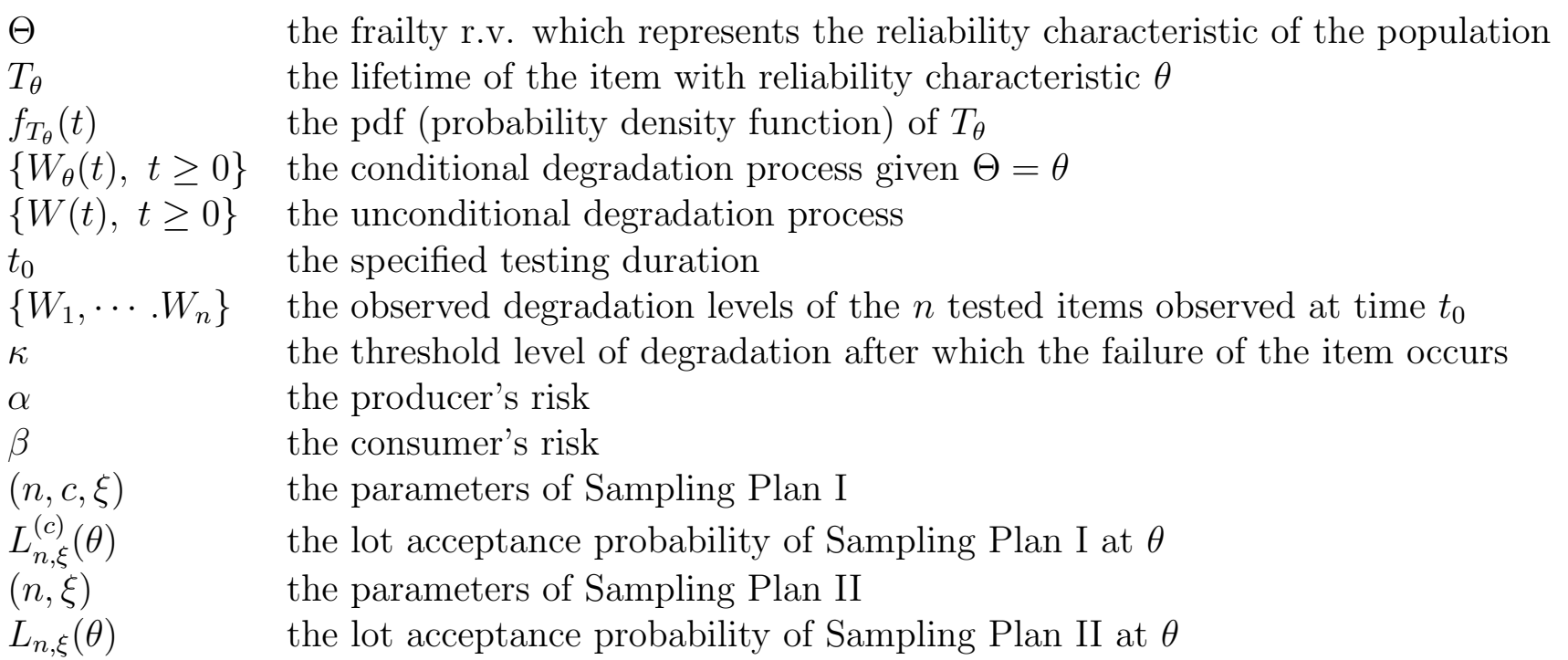

\section{Introduction}

In an acceptance sampling plan, the decision on whether to accept or reject a lot of items is based on the observation of a random sample drawn from the lot. One can refer to Stephens (2001) and Montgomery (2012) for general introductions to acceptance sampling plans. In the specific case where the 'lifetime' of a product is the main characteristic of interest, sampling plans designed for testing the acceptability of a product are called life test reliability sampling plans. Based on the observation of the lifetimes of the items on tests or on the number of failures observed during a pre-specified testing time, a decision to reject or accept the corresponding lot is reached, taking into account both the producer's and consumer's risks. See, for example, Epstein (1954), Epstein and Sobel (1953, 1955), Blugren and Hewette (1973), Fairbanks (1988), Fertig and Mann (1980) and Schneider (1989) for different acceptance reliability sampling plans for exponential or Weibull distributions. Later on, more sophisticated reliability sampling plans have been developed. See, for instance, Balakrishnan et al. (1993, 2007), Aslam and Jun (2009) and Aslam et al. (2011a, 2011b, 2013a, 2013b, 2013c), Chen et al. (2004), Tsai and Wanbo (2009), Fernández et al. (2011), Wu et al. (2018). Recently, Cha (2016) has addressed the effect of acceptance sampling tests on the reliability performance of the items accepted in the test. Cha (2015) and Lee and Cha (2017) have also studied reliability sampling plans for repairable items.

However, frequently in practice, items are subject to degradation phenomena. See Cha and Badía (2020a, 2020b) for detailed examples of the light intensity of an optical item and the electric voltage or current of some electrical items. Pan et al. (2016, 2017) have studied reliability estimation approach based on a Wiener degradation process and an inverse Gaussian degradation process with random effect, respectively. See also Van Noortwijk (2009) for a review of the various applications of gamma degradation process to various reliability maintenance modeling. In such a case when items are subject to degradation phenomena, instead of observing items' lifetimes (which gen- 
erally takes very long time), the degradation levels of the items can be observed on degradation tests (which are performed during a relatively short time). Then, based on this information, the decision on whether to accept or reject the corresponding lot can be made. Recently, Cha and Badía (2020a,2020b) studied sampling plans for items whose degradation process follows the gamma process and the inverse Gaussian process, respectively. In practice, the Wiener process is also frequently applied to model degradation phenomena (see Section 2) and there have been a few works on sampling plan based on the Wiener process. In Tsai et al. (2015), assuming the Wiener process of degradation, an acceptance test is proposed which minimizes the asymptotic variance of the estimate of the optimal acceptance testing time under a cost constrain. As a related work, one can also refer to Jin and Matthews (2014), where, assuming the Wiener process of degradation, a degradation-based testing procedure is developed to test whether the reliability of the product satisfies the required index or not.

In this paper, differently from Tsai et al. (2015) and Jin and Matthews (2014), we develop acceptance sampling plans which balance the producer's and consumer's risks based on the information about the degradation levels of the items, assuming the Wiener process of degradation. Furthermore, in addition to the sampling plan suggested in Cha and Badía (2020a,2020b), another new type of sampling plan which is based on the number of items whose degradation levels exceed a pre-determined level is proposed. Then, these two sampling plans are compared both from a technical and economical points of view. Note that classical reliability sampling plans based on life testing require the observation of failures during the test. Then, due to high reliability performance and long lifetimes of modern items, such plans can be of no use in practice as they can lead to impractically long testing durations. An important merit of the deterioration-based sampling plans proposed in this paper is that they do not require to observe failures of items, so that they can be implemented in much shorter time periods than the classical ones based on life testing.

The structure of this paper is as follows. In Section 2, a new reliability sampling plan for items subject to degradation phenomena is developed. This sampling plan is based on the number of items whose degradation levels exceed a pre-determined level. The parameter values of the sampling plan are determined by taking into account both producer's and consumer's risks. In addition, a convenient and efficient procedure is suggested for their practical implementation. Furthermore, we analyze the effect of the sampling plan on the reliability performance of the items in the population which has passed the testing procedure. In Section 3, we consider another reliability sampling plan for items subject to degradation phenomena, which is based on the sum of wear amounts accumulated by all the items put into the test. Similar issues are discussed for this second type of sampling plan as for the first one. Furthermore, the two sampling plans proposed in this paper are compared. Finally, concluding remarks are given in Section 4. 


\section{Degradation-Based Sampling Plan I}

\subsection{Degradation Process and Sampling Plan}

We now start our main discussion and first describe how to characterize the reliability level of an item. Frequently, there is variability in the reliability level of an item and some possible causes for it are the quality of resources and components supplied for the production, human errors, uncontrollable factors affecting the quality of the item, and so on. For instance, poor quality of resources and components may result in a low reliability level of produced items, and vice versa.

In this paper, the reliability level of an item is described through an Euclidian parameter $\theta$ that we call reliability characteristic. The possible variability in this reliability characteristic is translated through the use of a r.v. $\Theta$, and we write $\Theta=\theta$ to indicate that the reliability characteristic is $\theta$.

The degradation process of an items is denoted by $\{W(t), t \geq 0\}$, where $W(t)$ is the total amount of accumulated degradation until time $t$. As mentioned earlier, this degradation process depends on the reliability characteristic $\theta$ and $\left\{W_{\theta}(t), t \geq 0\right\}$ denotes the degradation process of an item given that $\Theta=\theta$. That is, $\left\{W_{\theta}(t), t \geq 0\right\}$ is the corresponding conditional degradation process given $\Theta=\theta$ and $W_{\theta}(t)$ is the total amount of accumulated degradation until time $t$ given $\Theta=\theta$, i.e., $W_{\theta}(t)=(W(t) \mid \Theta=\theta)$. We assume that the failure of an item occurs if its accumulated degradation exceeds the predetermined failure threshold $\kappa$. In practice, items whose accumulated degradation exceeds this level, can still operate. However, in this case, the item is not usable any more and is defined to be in the failed state.

There is a huge literature devoted to the stochastic modeling of accumulated degradation, among which the most common models are gamma process (Van Noortwijk (2009)), inverse Gaussian process (Wang and Xu (2010)) and Wiener process (Doksum and Hoyland (1992)). As noted by Hu et al. (2015), the Wiener process model has been used for many applications in a variety of studies. For instance, it is used by Whitmore (1995) to analyse the degradation (gain) of a transistor, by Wang (2010) to model the deterioration of bridge beams due to chloride ion ingression, by Si et al. (2013) for the analysis of gyroscopic drift in an inertial navigation system, by Lu and Meeker (1993) to analyse the data from fatigue crack growth subject to loading cycles, to chose only a few among many other papers. We refer to Zhang et al. (2018) or Hu et al. (2015) for more examples and references. We also refer to Kahle et al. (2016) for some review on its use as a deterioration process and statistical inference. Based on the previous literature, Wiener process is an adequate and popular model for modeling deterioration. We here assume that the conditional degradation process $\left\{W_{\theta}(t), t \geq 0\right\}$ (given $\Theta=\theta$ ) follows a Wiener process with positive drift.

Specifically, in this paper, $W_{\theta}(t)$ follows the following Wiener process:

$$
W_{\theta}(t)=\mu(\theta) t+\sigma B_{t},
$$

where $\left(B_{t}\right)_{t \geq 0}$ is a Brownian motion and $\mu(\theta)$ is strictly increasing in $\theta$. Thus, $W_{\theta}(t) \sim$ $\mathcal{N}\left(\mu(\theta) t, \sigma^{2} t\right)$ and then, for example, $E\left[W_{\theta}(t)\right]=\mu(\theta) t$ is also monotonically increasing in $\theta$, for all $t>0$. Thus, it implies that the higher $\theta$ is, the higher the deterioration level 
is. The effect of $\theta$ on the lifetime of an item can be made more precise using stochastic ordering. For the definitions of the classical stochastic orders used in this paper (usual stochastic order: $\prec_{s t}$, hazard rate order: $\prec_{h r}$, reversed hazard rate order: $\prec_{r h}$, likelihood ratio order: $\prec_{l r}$ ), we refer to Müller and Stoyan (2002) and to Shaked and Shanthikumar (2007).

Let $T_{\theta}$ stand for the lifetime of an item with reliability characteristic $\theta$, which is defined as the first-passage time of $\left(W_{\theta}(t)\right)_{t \geq 0}$ over level $\kappa>0$, namely:

$$
T_{\theta}=\inf \left(t \geq 0: W_{\theta}(t) \geq \kappa\right) \text {. }
$$

It is known that $T_{\theta}$ is inverse Gaussian distributed with pdf

$$
f_{T_{\theta}}(t)=\frac{\kappa}{\sqrt{2 \pi \sigma^{2} t^{3}}} \exp \left(-\frac{(\kappa-\mu(\theta) t)^{2}}{2 \sigma^{2} t}\right), \forall t \geq 0
$$

(see, e.g., Folks and Chhikara (1978)).

The following result explains that the lifetime of an item is decreasing in $\theta$ in a strong stochastic sense.

Proposition $1 T_{\theta}$ decreases with respect to $\theta$ in the likelihood ratio ordering, i.e., $T_{\theta_{2}} \prec_{l r} T_{\theta_{1}}$, for all $\theta_{1}<\theta_{2}$.

Proof. For $\theta_{1}<\theta_{2}$, we have

$$
\begin{aligned}
& \frac{f_{T_{\theta_{1}}}(t)}{f_{T_{\theta_{2}}}(t)} \\
& =\exp \left(\frac{-\left(\kappa-\mu\left(\theta_{1}\right) t\right)^{2}+\left(\kappa-\mu\left(\theta_{2}\right) t\right)^{2}}{2 \sigma^{2} t}\right) \\
& =\exp \left(\frac{\mu\left(\theta_{2}\right)-\mu\left(\theta_{1}\right)}{2 \sigma^{2}}\left(t\left(\mu\left(\theta_{1}\right)+\mu\left(\theta_{2}\right)\right)-2 \kappa\right)\right) .
\end{aligned}
$$

As $\mu\left(\theta_{1}\right)<\mu\left(\theta_{2}\right)$, the previous ratio increases with respect to $t$. This implies that $T_{\theta_{2}} \prec_{l r} T_{\theta_{1}}$ and the result.

As the likelihood ratio ordering implies the hazard rate and usual stochastic ordering, Proposition 1 also implies the ordering of $T_{\theta_{1}}$ and $T_{\theta_{2}}$ in terms of the corresponding hazard rate and survival function (see Shaked and Shanthikumar (2007)). Hence, the highest the reliability characteristic $\theta$ is, the lowest the reliability function $R_{\theta}(t)=$ $\mathbb{P}\left(T_{\theta}>t\right)$ is (for all $t \geq 0$ ) and the lowest the mean time to failure $\mathbb{E}\left[T_{\theta}\right]$ is.

As explained in the beginning of this section, the reliability characteristic of an item depends on several features such as the quality of resources and components used in the production process, among others. Typically, such features are common to all the units produced in a same lot. Hence, although the reliability characteristic of produced items is variable, we here assume that all the items belonging to a same lot are produced by a sufficiently stable manufacturing process so that they share the same reliability 
characteristic $\theta$, and hence, the same degradation process $\left\{W_{\theta}(t), t \geq 0\right\}$. Also, as usual, the items are assumed to be conditionally identical and independent in a lot, given that their common reliability characteristic is $\theta$ (namely given $\Theta=\theta$ ).

We are now interested in designing a sampling plan in order to ensure that the mean time to failure of an item is greater than a certain level. Based on the previously observed monotonicity of $\mathbb{E}\left[T_{\theta}\right]$ with respect to $\theta$, the point is to propose a plan which ensures that the reliability characteristic $\theta$ is smaller than a certain level. In such a plan, the probability of rejecting a good lot is called the producer's risk, whereas the probability of accepting a bad lot is known as the consumer's risk. Let us introduce two bounds $\theta_{i}, i=1,2$, where $\theta_{1}>\theta_{2}$, so that $\theta_{1}$ and $\theta_{2}$ stand for high and low quality bounds, respectively. The consumer demands that the lot acceptance probability should be smaller than the specified consumer's risk $\beta$ when testing a lot with a low quality level $\theta \leq \theta_{2}$, whereas the producer requires that the lot rejection probability should be smaller than the specified producer's risk $\alpha$, in case of a lot with a high quality level $\theta \geq \theta_{1}$ (where $\alpha, \beta \in(0,1)$ ). Based again on the monotonicity of $\mathbb{E}\left[T_{\theta}\right]$ with respect to $\theta$, it is equivalent to design the plan such that the lot acceptance probability is $\beta$ when testing a lot with quality level $\theta_{2}$ (consumer's risk) and that the lot rejection probability is $\alpha$, in case of a lot with quality level $\theta_{1}$ (producer's risk).

From the lot to be tested, $n$ items are randomly selected and tested for a time interval $t_{0}$, where $t_{0}$ is a specified testing duration. Let $W_{1}, W_{2}, \cdots, W_{n}$ stand for the degradation levels of these $n$ items observed at time $t_{0}$. Remembering that a larger value of $\theta$ (lower quality level) results in a larger value of $W_{\theta}(t)$, a possible acceptance test is as follows.

Rejection Rule of Sampling Plan I: If $\sum_{i=1}^{n} I\left(W_{i}>\xi\right)>c$, then the lot is rejected; otherwise the lot is accepted, where $\xi>0$ and $c \in\{0,1,2, \cdots, n-1\}$ are pre-determined parameters and $I(\cdot)$ is the indicator function, that is, $I(A)=1$ if condition $A$ is satisfied; $I(A)=0$, otherwise.

Thus, by Plan I, the lot is rejected if there are strictly more than $c$ items whose degradation level is larger that $\xi$ and the proposed sampling plan is characterized by three parameters $(n, c, \xi)$. For $n \geq 1, c \in\{0, \cdots, n-1\}$ and $\xi>0$, the acceptance probability of a lot as a function of $\theta$ is given by

$$
\begin{aligned}
L_{n, \xi}^{(c)}(\theta) & =\mathbb{P}\left(\sum_{i=1}^{n} I\left(W_{i}>\xi\right) \leq c\right) \\
& =\sum_{i=0}^{c}\left(\begin{array}{c}
n \\
i
\end{array}\right)\left(\bar{F}_{\theta}(\xi)\right)^{i}\left(F_{\theta}(\xi)\right)^{n-i},
\end{aligned}
$$

where $\bar{F}_{\theta}(\xi)=\mathbb{P}\left(W_{\theta}\left(t_{0}\right)>\xi\right)$ and $F_{\theta}(\xi)=1-\bar{F}_{\theta}(\xi)$.

It is now necessary to determine the parameters $(n, c, \xi)$ so that the consumer's risk and the producer's risk are balanced as follows:

$$
L_{n, \xi}^{(c)}\left(\theta_{2}\right)=\beta \text { and } 1-L_{n, \xi}^{(c)}\left(\theta_{1}\right)=\alpha .
$$

The following result allows us to understand the influence of each parameter on the 
acceptance probability $L_{n, \xi}^{(c)}(\theta)$ and to construct an efficient procedure in determining parameters (approximately) satisfying the two equations in (1).

Proposition 2 With all parameters fixed except from one, $L_{n, \xi}^{(c)}(\theta)$ increases with respect to $\xi$ and $c$, and decreases with respect to $n$ and $\theta$.

Proof. Increasingness of $L_{n, \xi}^{(c)}(\theta)$ with respect to $c$ is clear. Also, based on Cha (2016), a binomial random variable (r.v.) $\mathcal{B}(n, p)$ is known to increase in the likelihood ratio ordering with respect to $n$, and hence also in the usual stochastic ordering. Then $L_{n, \xi}^{(c)}(\theta)$ decreases with respect to $n$. Based on Klenke and Mattner $(2010), \mathcal{B}(n, p)$ increases in the usual stochastic ordering when $p$ increases. Also, based on the fact that $W_{\theta}\left(t_{0}\right)$ increases with respect to $\theta$ in the likelihood ratio sense (see Müller and Stoyan (2002), page 62$), W_{\theta}\left(t_{0}\right)$ also increases in the usual stochastic order sense, so that $\bar{F}_{\theta}(\xi)$ increases with respect to $\theta$ and $L_{n, \xi}^{(c)}(\theta)$ decreases with respect to $\theta$. Increasingness of $L_{n, \xi}^{(c)}(\theta)$ with respect to $\xi$ is proved in a similar way.

Suppose that we search for $(n, c)$ satisfying (1) for a fixed $\xi$. Considering successive $n=1,2, \ldots$, we first look for $c$ such that

$$
L_{n, \xi}^{(c)}\left(\theta_{1}\right)=1-\alpha .
$$

Denote this value of $c$ by $c(n)$. If this $c(n)$ does not satisfy

$$
L_{n, \xi}^{(c)}\left(\theta_{2}\right)=\beta,
$$

then we fix the parameter of the number of tested items as $n+1$ and look for the corresponding $c(n+1)$, and so on. Due to Proposition 2, we already know that $c(n) \leq$ $c(n+1), n=1,2, \cdots$. Thus, $c(n)$ is a lower bound for $c(n+1)$, which limits the range for $c(n+1)$ to $\{c(n), \cdots, n\}$.

In some cases, there cannot be exact values of $(n, c, \xi)$ satisfying the two equations in (1). Thus, we take $(n, c, \xi)$ which achieves the nearest acceptance probabilities:

$$
L_{n, \xi}^{(c)}\left(\theta_{2}\right) \approx \beta
$$

and

$$
1-L_{n, \xi}^{(c)}\left(\theta_{1}\right) \approx \alpha
$$

or equivalently

$$
L_{n, \xi}^{(c)}\left(\theta_{1}\right) \approx 1-\alpha
$$

To find the parameters satisfying (2) and (3), the following algorithm is used, where $n_{\max }$ is the maximum allowable sample size and $\varepsilon>0$ is a precision threshold, which should be fixed in advance. 


\section{Algorithm 1}

1. Fix $\xi$

2. Initialization for $n=1$ :

(a) Set $c(1)=0$;

(b) If $\left|L_{1, \xi}^{(0)}\left(\theta_{1}\right)-1+\alpha\right| \leq \varepsilon$ and $\left|L_{1, \xi}^{(0)}\left(\theta_{2}\right)-\beta\right| \leq \varepsilon$, store the value $(n=1, c(1)=0)$;

3. For each $2 \leq n \leq n_{\max }$ :

(a) Compute $L_{n, \xi}^{(c)}\left(\theta_{1}\right)$ for each $c \in\{c(n-1), \cdots, n-1\}$;

(b) Find $c(n)$ which minimizes $\left|L_{n, \xi}^{(c)}\left(\theta_{1}\right)-1+\alpha\right|$ as a function of $c$;

(c) If $\left|L_{n, \xi}^{(c(n))}\left(\theta_{1}\right)-1+\alpha\right| \leq \varepsilon$ and $\left|L_{n, \xi}^{(c(n))}\left(\theta_{2}\right)-\beta\right| \leq \varepsilon$, then store the value of $(n, c(n))$;

4. Among all $(n, c(n))$ stored in the previous stages, find the smallest $n$.

For each $\xi$, the above algorithm allows us to obtain the corresponding $(n(\xi), c(\xi))$ such that $\left|L_{n(\xi), \xi}^{(c(\xi)(n))}\left(\theta_{1}\right)-1+\alpha\right| \leq \varepsilon$ and $\left|L_{n(\xi), \xi}^{(c(\xi)(n))}\left(\theta_{2}\right)-\beta\right| \leq \varepsilon$, namely such that $L_{n(\xi), \xi}^{(c(\xi))}\left(\theta_{1}\right) \simeq 1-\alpha$ and $L_{n(\xi), \xi}^{(c(\xi))}\left(\theta_{2}\right) \simeq \beta$. Next, we consider various $\xi$ in $\left(0, \xi_{0}\right]$ (where $\xi_{0}$ is a maximal threshold fixed in advance) and for each $\xi \in\left(0, \xi_{0}\right]$, we search for the optimal $\xi\left(\xi_{\text {opt }}\right.$, say) which minimizes $n(\xi)$. Then the obtained solution $\left(\xi_{\text {opt }}, n\left(\xi_{\text {opt }}\right), c\left(\xi_{\text {opt }}\right)\right)$ satisfies (2) and (3). Also the solution minimizes the total number of items used in the test, and hence minimizes the corresponding cost.

Example 1 Let $\theta_{1}=1 ; \theta_{2}=1.5 ; t_{0}=1 ; \mu(\theta)=\theta ; \sigma=0.5 ; \alpha=0.05 ; \beta=0.1 ; n_{\max }=30$ and $\xi \in[0.01,2]$. Using Algorithm 1, the corresponding $n(\xi), c(\xi), R_{1}(\xi), R_{2}(\xi)$ are obtained and plotted with respect to $\xi$ in Figure 1, where

$$
\begin{aligned}
& R_{1}(\xi)=L_{n(\xi), \xi}^{(c(\xi))}\left(\theta_{1}\right)-1+\alpha, \\
& R_{2}(\xi)=\beta-L_{n(\xi), \xi}^{(c(\xi))}\left(\theta_{2}\right) .
\end{aligned}
$$

For $\varepsilon=0.01$ we obtain: $\xi_{\text {opt }}=1.23, n\left(\xi_{\text {opt }}\right)=12, c\left(\xi_{\text {opt }}\right)=6, R_{1}\left(\xi_{\text {opt }}\right)=-6.4 \times 10^{-3}$ and $R_{2}\left(\xi_{\text {opt }}\right)=-9.5 \times 10^{-3}$. The value of $\xi_{\text {opt }}$ is highlighted with a red dotted line in Figure 1 . As can be seen in the figure, the procedure chooses $\xi_{\text {opt }}$ which minimizes $n\left(\xi_{\text {opt }}\right)$ under the condition that $R_{1}\left(\xi_{\text {opt }}\right)$ and $R_{2}\left(\xi_{\text {opt }}\right)$ are near zero.

Example 2 We keep all the previous parameters except for $\theta_{2}, \sigma$ and $\varepsilon$ (and $n_{\max }$ ), for which several values are envisioned. The results are provided in Table 1 for $\sigma=0.5$ (and $n_{\max }=55$ ), in Table 2 for $\sigma=0.75$ (and $n_{\max }=100$ ) and in Table 3 for $\sigma=0.25$ (and $n_{\max }=20$ ), where, in each case, $\varepsilon$ has been mostly adjusted to get the best precision and $n_{\max }$ is chosen such that $\xi_{\text {opt }}$ exists, which complies with the conditions given in Algorithm 1. 

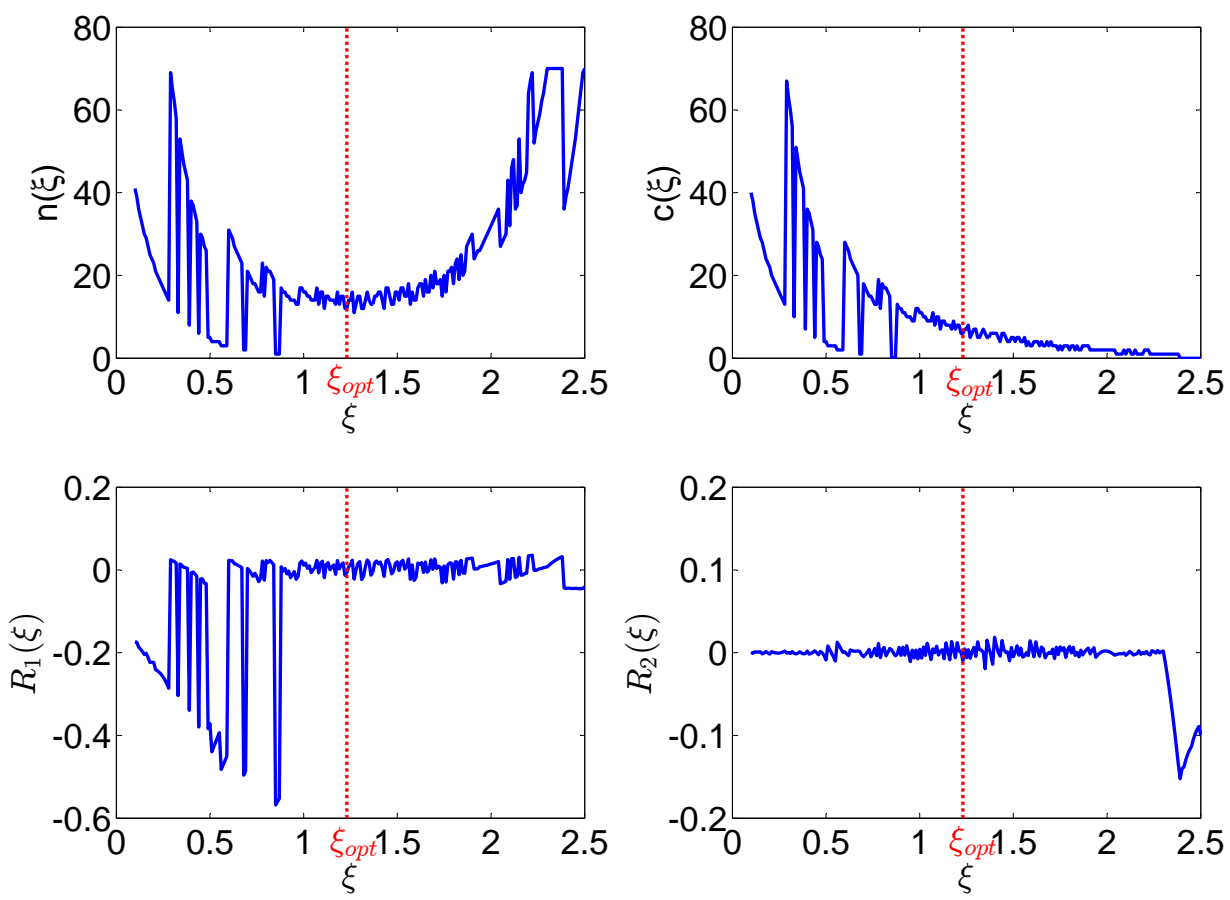

Figure 1: $n(\xi), c(\xi), R_{1}(\xi), R_{2}(\xi)$ with respect to $\xi$ together with $\xi_{\text {opt }}$, Example 1

\begin{tabular}{cccccrr}
\hline$\theta_{2}$ & $\varepsilon$ & $\xi_{\text {opt }}$ & $n\left(\xi_{\text {opt }}\right)$ & $c\left(\xi_{\text {opt }}\right)$ & $R_{1}\left(\xi_{\text {opt }}\right)$ & $R_{2}\left(\xi_{\text {opt }}\right)$ \\
\hline 1.25 & 0.01 & 1.10 & 50 & 26 & $-9.6 \times 10^{-3}$ & $-1.4 \times 10^{-3}$ \\
1.50 & 0.01 & 1.23 & 12 & 6 & $-6.4 \times 10^{-3}$ & $-9.5 \times 10^{-3}$ \\
1.75 & 0.01 & 1.42 & 5 & 2 & $-8.3 \times 10^{-3}$ & $-8.5 \times 10^{-3}$ \\
2 & 0.01 & 1.56 & 3 & 1 & $2.8 \times 10^{-3}$ & $5.9 \times 10^{-3}$ \\
2.25 & 0.01 & 1.41 & 2 & 1 & $7.5 \times 10^{-3}$ & $9.2 \times 10^{-3}$ \\
2.50 & 0.01 & 1.83 & 1 & 0 & $1.5 \times 10^{-3}$ & $9.9 \times 10^{-3}$ \\
2.75 & 0.1 & 1.83 & 1 & 0 & $1.5 \times 10^{-3}$ & $6.7 \times 10^{-2}$ \\
3 & 0.1 & 1.83 & 1 & 0 & $1.5 \times 10^{-3}$ & $9.0 \times 10^{-2}$ \\
4 & 0.1 & 1.83 & 1 & 0 & $1.5 \times 10^{-3}$ & $1.0 \times 10^{-1}$ \\
\hline
\end{tabular}

Table 1: $\xi_{\text {opt }}, n\left(\xi_{\text {opt }}\right), c\left(\xi_{\text {opt }}\right), R_{1}\left(\xi_{\text {opt }}\right), R_{2}\left(\xi_{\text {opt }}\right)$ with respect to $\theta_{2}$, Example 2, case $\sigma=0.5\left(\right.$ and $\left.n_{\max }=55\right)$

From Tables 1, 2 and 3, we find that $n\left(\xi_{\text {opt }}\right)$ decreases as $\theta_{2}$ increases (except for the last line of Table 2), namely, when there is a larger difference between $\theta_{1}$ and $\theta_{2}$. Indeed, it seems natural that less units are required to make a distinction between the two levels when they are well separate. Furthermore, we observe that $c\left(\xi_{\text {opt }}\right)$ decreases when $\theta_{2}$ increases (except for the last line of Table 2). Also, from $\theta_{2} \simeq 2.50$, the values of $\xi_{\text {opt }}$, $n\left(\xi_{\text {opt }}\right), c\left(\xi_{\text {opt }}\right)$ and $R_{1}\left(\xi_{\text {opt }}\right)$ become stable. However, $R_{2}\left(\xi_{\text {opt }}\right)$ increases and, for large values of $\theta_{2}$, it becomes more difficult to comply with the constraint $R_{2}\left(\xi_{\text {opt }}\right) \simeq 0$. For a better understanding of the behavior of the procedure for large $\theta_{2}$, we refer to Figure 


\begin{tabular}{cccccrr}
\hline$\theta_{2}$ & $\varepsilon$ & $\xi_{\text {opt }}$ & $n\left(\xi_{\text {opt }}\right)$ & $c\left(\xi_{\text {opt }}\right)$ & $R_{1}\left(\xi_{\text {opt }}\right)$ & $R_{2}\left(\xi_{\text {opt }}\right)$ \\
\hline 1.25 & 0.05 & 1.09 & 99 & 52 & $-9.6 \times 10^{-3}$ & $-3.7 \times 10^{-2}$ \\
1.50 & 0.05 & 1.13 & 27 & 15 & $-1.8 \times 10^{-2}$ & $-5.0 \times 10^{-4}$ \\
1.75 & 0.05 & 1.40 & 11 & 5 & $-2.5 \times 10^{-2}$ & $-3.6 \times 10^{-3}$ \\
2 & 0.05 & 1.36 & 6 & 3 & $-3.4 \times 10^{-2}$ & $5.1 \times 10^{-3}$ \\
2.25 & 0.05 & 1.67 & 3 & 1 & $-4.1 \times 10^{-2}$ & $-2.4 \times 10^{-2}$ \\
2.50 & 0.1 & 1.77 & 3 & 1 & $-1.3 \times 10^{-2}$ & $2.7 \times 10^{-2}$ \\
2.75 & 0.1 & 1.78 & 1 & 0 & $-9.9 \times 10^{-2}$ & $2.1 \times 10^{-3}$ \\
3 & 0.1 & 1.89 & 1 & 0 & $-6.8 \times 10^{-2}$ & $3.1 \times 10^{-2}$ \\
4 & 0.1 & 1.67 & 2 & 1 & $1.6 \times 10^{-2}$ & $9.8 \times 10^{-2}$ \\
\hline
\end{tabular}

Table 2: $\xi_{\text {opt }}, n\left(\xi_{\text {opt }}\right), c\left(\xi_{\text {opt }}\right), R_{1}\left(\xi_{\text {opt }}\right), R_{2}\left(\xi_{\text {opt }}\right)$ with respect to $\theta_{2}$, Example 2, case $\sigma=0.75\left(\right.$ and $\left.n_{\max }=100\right)$

\begin{tabular}{cccccrr}
\hline$\theta_{2}$ & $\varepsilon$ & $\xi_{\text {opt }}$ & $n\left(\xi_{\text {opt }}\right)$ & $c\left(\xi_{\text {opt }}\right)$ & $R_{1}\left(\xi_{\text {opt }}\right)$ & $R_{2}\left(\xi_{\text {opt }}\right)$ \\
\hline 1.25 & 0.05 & 1.11 & 12 & 6 & $-1.3 \times 10^{-2}$ & $6.0 \times 10^{-4}$ \\
1.35 & 0.05 & 1.19 & 2 & 1 & $-2.8 \times 10^{-2}$ & $-1.6 \times 10^{-2}$ \\
1.45 & 0.05 & 1.23 & 1 & 0 & $-3.5 \times 10^{-2}$ & $5.9 \times 10^{-3}$ \\
1.75 & 0.05 & 1.38 & 1 & 0 & $-1.4 \times 10^{-2}$ & $3.1 \times 10^{-2}$ \\
2.00 & 0.1 & 1.59 & 1 & 0 & $4.1 \times 10^{-2}$ & $4.9 \times 10^{-2}$ \\
2.25 & 0.1 & 1.42 & 1 & 0 & $3.5 \times 10^{-2}$ & $9.9 \times 10^{-2}$ \\
2.5 & 0.1 & 1.42 & 1 & 0 & $3.5 \times 10^{-2}$ & $1.0 \times 10^{-1}$ \\
3 & 0.1 & 1.42 & 1 & 0 & $3.5 \times 10^{-2}$ & $1.0 \times 10^{-1}$ \\
\hline
\end{tabular}

Table 3: $\xi_{\text {opt }}, n\left(\xi_{\text {opt }}\right), c\left(\xi_{\text {opt }}\right), R_{1}\left(\xi_{\text {opt }}\right), R_{2}\left(\xi_{\text {opt }}\right)$ with respect to $\theta_{2}$, Example 2, case $\sigma=0.25\left(\right.$ and $\left.n_{\max }=20\right)$

2 , which corresponds to $\theta_{2}=4$. We can see in that figure that, indeed, when $\theta_{2}$ is large in front of $\theta_{1}$, it becomes difficult to comply with the two constraints. However, in that case, we have $L_{n(\xi), \xi}^{(c(\xi))}\left(\theta_{1}\right) \approx 1-\alpha$ and $L_{n(\xi), \xi}^{(c(\xi))}\left(\theta_{2}\right)<\beta$ and the aim of the sampling plan is still fulfilled, with an even lower consumer's risk than required.

\subsection{Analysis of Reliability Characteristic of an Accepted Lot}

In this section, we investigate the effect of the sampling plan developed in the previous subsection on the reliability characteristic of an item belonging to a lot which has been accepted by the testing procedure. Before discussing the lifetime of the item, we first study its amount of degradation in an arbitrary time interval $W\left(t_{2}\right)-W\left(t_{1}\right), t_{1}<t_{2}$. As stated before, the reliability characteristic of the population is variable due to many different reasons and the variability is represented by the r.v. $\Theta$. We set $f_{\Theta}$ to be its pdf. Before the testing procedure, the distribution of the increment $W\left(t_{2}\right)-W\left(t_{1}\right)$ is a mixed distribution with survival function

$$
\bar{F}_{t_{1}, t_{2}}(x)=\int_{\mathbb{R}_{+}} \bar{F}_{t_{1}, t_{2}, \theta}(x) f_{\Theta}(\theta) d \theta, \forall x \geq 0
$$



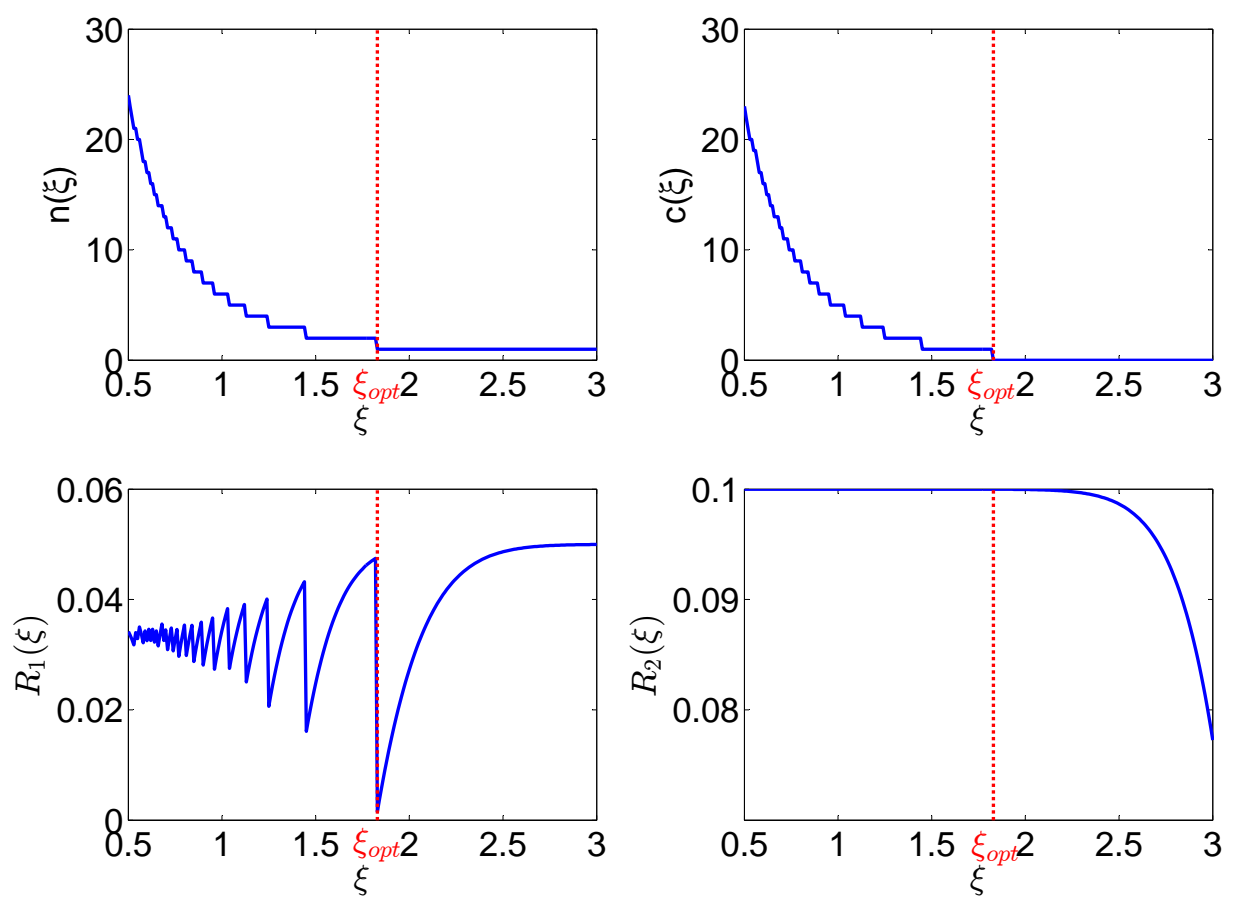

Figure 2: $n(\xi), c(\xi), R_{1}(\xi), R_{2}(\xi)$ with respect to $\xi$ together with $\xi_{\text {opt }}$, case $\theta_{2}=4$, Example 2

and pdf:

$$
f_{t_{1}, t_{2}}(x)=\int_{\mathbb{R}_{+}} f_{t_{1}, t_{2}, \theta}(x) f_{\Theta}(\theta) d \theta, \forall x \geq 0,
$$

where $\bar{F}_{t_{1}, t_{2}, \theta}$ and and $f_{t_{1}, t_{2}, \theta}$ are the survival function and the pdf of $W_{\theta}\left(t_{2}\right)-W_{\theta}\left(t_{1}\right)$, respectively, which is Gaussian distributed $\mathcal{N}\left(\mu(\theta)\left(t_{2}-t_{1}\right), \sigma^{2}\left(t_{2}-t_{1}\right)\right)$.

For $0 \leq t_{1}<t_{2}$, let $\bar{F}_{t_{1}, t_{2} \mid S}$ be the conditional survival function of $W\left(t_{2}\right)-W\left(t_{1}\right)$ for a randomly chosen item given that the test with the parameters $(\xi, n, c)$ was successful (i.e., given that the lot is accepted), where $S=\left\{\sum_{i=1}^{n} I\left(W_{i}>\xi\right)>c\right\}$. Then we obtain the following result.

Proposition 3 For all $0 \leq t_{1}<t_{2}$, we have:

$$
\begin{gathered}
\bar{F}_{t_{1}, t_{2} \mid S}(x)=\frac{\int_{\mathbb{R}_{+}} \bar{F}_{t_{1}, t_{2}, \theta}(x) L_{n, \xi}^{(c)}(\theta) f_{\Theta}(\theta) d \theta}{\int_{\mathbb{R}_{+}} L_{n, \xi}^{(c)}(\theta) f_{\Theta}(\theta) d \theta}, \forall x \geq 0, \\
f_{t_{1}, t_{2} \mid S}(x)=\frac{\int_{\mathbb{R}_{+}} f_{t_{1}, t_{2}, \theta}(x) L_{n, \xi}^{(c)}(\theta) f_{\Theta}(\theta) d \theta}{\int_{\mathbb{R}_{+}} L_{n, \xi}^{(c)}(\theta) f_{\Theta}(\theta) d \theta}, \forall x \geq 0 .
\end{gathered}
$$


Proof. Let $f_{\Theta \mid S}(\theta)$ be the conditional pdf of $\Theta \mid S$. We have:

$$
\begin{aligned}
\bar{F}_{t_{1}, t_{2} \mid S}(x) & =\int_{\mathbb{R}_{+}} \bar{F}_{t_{1}, t_{2}, \theta}(x) f_{\Theta \mid S}(\theta) d \theta, \\
f_{t_{1}, t_{2} \mid S}(x) & =\int_{\mathbb{R}_{+}} f_{t_{1}, t_{2}, \theta}(x) f_{\Theta \mid S}(\theta) d \theta,
\end{aligned}
$$

where

$$
\begin{aligned}
f_{\Theta \mid S}(\theta) & =f_{\Theta \mid \sum_{i=1}^{n} I\left(W_{i}>\xi\right)>c}(\theta) \\
& =\frac{\mathbb{P}\left(\sum_{i=1}^{n} I\left(W_{i}>\xi\right)>c \mid \Theta=\theta\right) f_{\Theta}(\theta)}{\mathbb{P}\left(\sum_{i=1}^{n} I\left(W_{i}>\xi\right)>c\right)} \\
& =\frac{L_{n, \xi}^{(c)}(\theta) f_{\Theta}(\theta)}{\int_{\mathbb{R}^{+}} L_{n, \xi}^{(c)}(\theta) f_{\Theta}(\theta) d \theta}
\end{aligned}
$$

which provides the two results.

The following result stochastically compares the r.v. $\Theta$ and $W\left(t_{2}\right)-W\left(t_{1}\right)$ before and after the testing procedure.

Proposition 4 It holds that

1. $[\Theta \mid S] \prec_{l r} \Theta$;

2. $\left[W\left(t_{2}\right)-W\left(t_{1}\right) \mid S\right] \prec_{l r} W\left(t_{2}\right)-W\left(t_{1}\right)$, for all $0 \leq t_{1}<t_{2}$.

Proof. We have

$$
\frac{f_{\Theta \mid S}(\theta)}{f_{\Theta}(\theta)}=C L_{n, \xi}^{(c)}(\theta)
$$

(where $C$ is a constant which does not depend on $\theta$ ) and the ratio is decreasing with respect to $\theta$ (see Proposition 2). This provides the first point.

As for the second point, we have

$$
\begin{aligned}
f_{t_{1}, t_{2}}(x) & =\int_{\mathbb{R}_{+}} f_{t_{1}, t_{2}, \theta}(x) f_{\Theta}(\theta) d \theta, \\
f_{t_{1}, t_{2} \mid S}(x) & =\int_{\mathbb{R}_{+}} f_{t_{1}, t_{2}, \theta}(x) f_{\Theta \mid S}(\theta) d \theta .
\end{aligned}
$$

Also, we know from Müller and Stoyan (2002), page 62, that $W_{\theta}\left(t_{2}\right)-W_{\theta}\left(t_{1}\right)$ increases with respect to $\theta$ in the likelihood ratio order sense. As $[\Theta \mid S] \prec_{l r} \Theta$ from the first point, the second point now follows from Shaked and Shanthikumar (2007) (page 49, Theorem 1.C.17).

Obviously, before the testing procedure, the distribution of the first-passage time $T$ of $W(t)$ over level $\kappa>0$ of a randomly chosen item is a mixed distribution with pdf:

$$
f_{T}(x)=\int_{\mathbb{R}_{+}} f_{T_{\theta}}(x) f_{\Theta}(\theta) d \theta, \forall x \geq 0 .
$$


Now, given that the test was successful (i.e., the lot is accepted), the conditional pdf of $T$ given $S=\left\{\sum_{i=1}^{n} I\left(W_{i}>\xi\right)>c\right\}$ becomes

$$
f_{T \mid S}(x)=\int_{\mathbb{R}_{+}} f_{T_{\theta}}(x) f_{\Theta \mid S}(\theta) d \theta, \forall x \geq 0 .
$$

In the following result, we compare the lifetimes before and after the testing procedure.

Proposition 5 It holds that $T \prec_{l r}[T \mid S]$.

Proof. Based on Proposition 1 and Shaked and Shanthikumar (2007) (page 46, Theorem 1.C.8), $-T_{\theta}$ increases with respect to $\theta$ in the likelihood ratio ordering. Using the first point of Proposition 4 and Shaked and Shanthikumar (2007) (page 49, Theorem 1.C.17), we derive that $[-T \mid S] \prec_{l r}-T$, which provides the result.

The above result entails for instance that $T \prec_{h r}[T \mid S]$, which means that the residual life of an item at any time $t$ is stochastically larger if we know that the lot has been accepted:

$$
[T-t \mid T>t] \prec_{\text {sto }}[T-t \mid S, T>t], \forall t \geq 0 .
$$

The above result also entails for instance that the mean residual life of an item at any time $t$ is larger if we know that the lot has been accepted:

$$
\begin{aligned}
M R L_{T}(t) & \equiv \mathbb{E}(T-t \mid T>t) \\
& \leq \mathbb{E}(T-t \mid S, T>t) \\
& \equiv M R L_{T \mid S}(t)
\end{aligned}
$$

for all $t \geq 0$.

\section{Degradation-Based Sampling Plan II}

\subsection{Sampling Plan}

The second type of sampling plan based on the degradation tests is as follows. As in the previous section, $n$ items are randomly chosen from the lot to be tested and tested for a time interval $t_{0}$. From the test, the values of the degradation levels of these $n$ items $W_{1}, W_{2}, \cdots, W_{n}$ are observed.

Rejection Rule of Sampling Plan II: If $\sum_{i=1}^{n} W_{i}>\xi$, then the lot is rejected; otherwise the lot is accepted, where $\xi>0$ is a pre-determined parameter.

Then the proposed sampling plan is characterized by two parameters $(n, \xi)$. Note that 
$\sum_{i=1}^{n} W_{i} \sim \mathcal{N}\left(n \mu(\theta) t_{0}, n \sigma^{2} t_{0}\right)$. Then, for $n \geq 1$ and $\xi>0$, the acceptance probability of the lot as a function of $\theta$ is given by

$$
L_{n, \xi}(\theta)=\mathbb{P}\left(\sum_{i=1}^{n} W_{i} \leq \xi\right)=F_{n, \theta}(\xi)
$$

where $F_{n, \theta}$ is the Cdf (cumulative distribution function) $\mathcal{N}\left(n \mu(\theta) t_{0}, n \sigma^{2} t_{0}\right)$.

As before, it is now necessary to determine the parameters $(n, \xi)$ so that the consumer's risk and the producer's risk are balanced as follows:

$$
L_{n, \xi}\left(\theta_{2}\right) \approx \beta, \text { and } L_{n, \xi}\left(\theta_{1}\right) \approx 1-\alpha .
$$

The following is the basic properties of the function $L_{n, \xi}(\theta)$.

Proposition 6 With all parameters fixed except from one, $L_{n, \xi}(\theta)$ increases with respect to $\xi$, and decreases with respect to $\theta$.

Proof. The fact that $L_{n, \xi}(\theta)$ increases with respect to $\xi$ is clear.

As $\sum_{i=1}^{n} W_{i}$ increases with respect to $\theta$ in the likelihood ratio order sense (see Müller and Stoyan (2002), page 62$), W_{\theta}\left(t_{0}\right)$ also increases in the usual stochastic order sense, so that $L_{n, \xi}(\theta)$ decreases with respect to $\theta$.

Remark 1 From the literature (see, e.g., Müller and Stoyan (2002), page 62), it is known that a Gaussian r.v. $\mathcal{N}\left(m, \sigma^{2}\right)$ increases when $(m, \sigma)$ increases in the increasing convex ordering, but not in the (stronger) usual stochastic ordering. Then, there is no reason why $\sum_{i=1}^{n} W_{i} \sim \mathcal{N}\left(n \mu(\theta) t_{0}, n \sigma^{2} t_{0}\right)$ should be monotone with respect to $n$ in the usual stochastic ordering and hence, there is no reason why $L_{n, \xi}(\theta)$ should be monotone with respect to $n$.

Note that, using the Cdf of the normal distribution, for a fixed $n$, the parameter $\xi$ satisfying $L_{n, \xi}\left(\theta_{1}\right)=1-\alpha$ can be easily obtained as $\xi=F_{n, \theta_{1}}^{-1}(1-\alpha)$. As before, to find the parameters $(n, \xi)$, the following algorithm is used, where $n_{\max }$ is the maximum allowable sample size and $\varepsilon>0$ is the threshold for the precision, which should be fixed in advance.

\section{Algorithm 2}

1. For each $1 \leq n \leq n_{\max }$ :

(a) Compute $\xi(n)=F_{n, \theta_{1}}^{-1}(1-\alpha)$;

(b) Compute $L_{n, \xi(n)}\left(\theta_{2}\right)$;

2. Chose the smallest $n$ such that $\left|L_{n, \xi(n)}\left(\theta_{2}\right)-\beta\right| \leq \varepsilon$.

The above algorithm allows us to obtain the corresponding optimal parameters $\left(n_{\text {opt }}, \xi\left(n_{\text {opt }}\right)\right)$ such that $n_{\text {opt }}$ is the minimum among all $n$ 's satisfying (4). Note that this algorithm is much simpler than Algorithm 1 for Sampling Plan I. 
Example 3 Let $\theta_{1}=1 ; t_{0}=1 ; \mu(\theta)=\theta ; \alpha=0.05 ; \beta=0.1$. Considering several values for $\theta_{2}, \sigma$ and $\varepsilon$ (and $\left.n_{\max }\right)$, the corresponding $\left(n_{\text {opt }}, \xi\left(n_{\text {opt }}\right)\right), R_{1}\left(n_{\text {opt }}\right), R_{2}\left(n_{\text {opt }}\right)$ are obtained using Algorithm 2. The results are provided in Table 4 for $\sigma=0.5$ (and $n_{\max }=50$ ), in Table 5 for $\sigma=0.75$ (and $n_{\max }=100$ ) and in Table 6 for $\sigma=0.25$ (and $\left.n_{\max }=10\right)$, where in each case, $\varepsilon$ has been mostly adjusted to get the best precision and $n_{\max }$ is chosen such that $\xi_{\text {opt }}$ exists, which complies with the conditions given in Algorithm 2.

From Tables 4, 5 and 6, we can see that, in the same way as for the first acceptance sampling plan, the larger $\theta_{2}$ is, the lower $n\left(\xi_{\text {opt }}\right)$ is. Here again, a larger difference between $\theta_{1}$ and $\theta_{2}$ hence requires less units to make the distinction between the two levels. Also, for each value of $\sigma$, the values of $\xi\left(n_{\text {opt }}\right)$ and $n_{\text {opt }}$ become stable from a certain value $\bar{\theta}_{2}$ for $\theta_{2}$. Note that the larger $\sigma$ is, the larger this threshold value $\bar{\theta}_{2}$ is, with $\bar{\theta}_{2} \approx 1.75$ for $\sigma=0.25, \bar{\theta}_{2} \approx 2.5$ for $\sigma=0.5$ and $\bar{\theta}_{2} \approx 3$ for $\sigma=0.75$. Such a remark is mostly valid for the first sampling plan as well, but the results are more stable and easy to interpret for the second one. Finally, here again, when $\theta_{2}$ is large in front of $\theta_{1}$,

we have $L_{n(\xi), \xi}^{(c(\xi))}\left(\theta_{1}\right) \approx 1-\alpha$ and $L_{n(\xi), \xi}^{(c(\xi))}\left(\theta_{2}\right)<\beta$, so that the aim of the sampling plan is still fulfilled.

\subsection{Analysis of Reliability Characteristic of an Accepted Lot}

As before, we first study the amount of degradation in an arbitrary time interval $W\left(t_{2}\right)-$ $W\left(t_{1}\right), t_{1}<t_{2}$. Let $S=\left\{\sum_{i=1}^{n} W_{i} \leq \xi\right\}$. We have a similar result as for Proposition 3 , substituting $L_{n, \xi}^{(c)(\theta)}$ by $L_{n, \xi}(\theta)$. The proof uses the same arguments and it is omitted.

Proposition 7 For all $0 \leq t_{1}<t_{2}$, we have:

$$
\begin{gathered}
\bar{F}_{t_{1}, t_{2} \mid S}(x)=\frac{\int_{\mathbb{R}_{+}} \bar{F}_{t_{1}, t_{2}, \theta}(x) L_{n, \xi}(\theta) f_{\Theta}(\theta) d \theta}{\int_{\mathbb{R}_{+}} L_{n, \xi}(\theta) f_{\Theta}(\theta) d \theta}, \forall x \geq 0, \\
f_{t_{1}, t_{2} \mid S}(x)=\frac{\int_{\mathbb{R}_{+}} f_{t_{1}, t_{2}, \theta}(x) L_{n, \xi}(\theta) f_{\Theta}(\theta) d \theta}{\int_{\mathbb{R}_{+}} L_{n, \xi}(\theta) f_{\Theta}(\theta) d \theta}, \forall x \geq 0 .
\end{gathered}
$$

Regarding the stochastic comparison of $\Theta$ and $W\left(t_{2}\right)-W\left(t_{1}\right)$ before and after the testing procedure, similar arguments as for Proposition 4 can be used to obtain the following result.

Proposition 8 It holds that

1. $[\Theta \mid S] \prec_{l r} \Theta$;

2. $\left[W\left(t_{2}\right)-W\left(t_{1}\right) \mid S\right] \prec_{l r} W\left(t_{2}\right)-W\left(t_{1}\right)$, for all $0 \leq t_{1}<t_{2}$.

Regarding the stochastic comparison of lifetimes, here again similar arguments as for Proposition 5 can be used to obtain the following result.

Proposition 9 It holds that $T \prec_{l r}[T \mid S]$.

As a conclusion, just as for Sampling Plan I, Sampling Plan II also allows us to know that the lifetime of an item belonging to an accepted lot is larger than if the lot had not been tested. 


\begin{tabular}{ccccrr}
\hline$\theta_{2}$ & $\varepsilon$ & $\xi\left(n_{\text {opt }}\right)$ & $n_{\text {opt }}$ & $R_{1}\left(n_{\text {opt }}\right)$ & $R_{2}\left(n_{\text {opt }}\right)$ \\
\hline 1.25 & 0.005 & 38.80 & 34 & $-4.16 \times 10^{-17}$ & $-2 \times 10^{-3}$ \\
1.50 & 0.025 & 10.33 & 8 & $-4.16 \times 10^{-17}$ & $-1.8 \times 10^{-2}$ \\
1.75 & 0.025 & 5.64 & 4 & $-4.16 \times 10^{-17}$ & $1.2 \times 10^{-2}$ \\
2 & 0.025 & 3.16 & 2 & $-4.16 \times 10^{-17}$ & $-1.8 \times 10^{-2}$ \\
2.25 & 0.075 & 3.16 & 2 & $-4.16 \times 10^{-17}$ & $7.1 \times 10^{-2}$ \\
2.50 & 0.075 & 1.82 & 1 & $-4.16 \times 10^{-17}$ & $1.2 \times 10^{-2}$ \\
2.75 & 0.075 & 1.82 & 1 & $-4.16 \times 10^{-17}$ & $6.8 \times 10^{-2}$ \\
3 & 0.1 & 1.82 & 1 & $-4.16 \times 10^{-17}$ & $9.1 \times 10^{-2}$ \\
4 & 0.1 & 1.82 & 1 & $-4.16 \times 10^{-17}$ & $1.0 \times 10^{-1}$ \\
\hline
\end{tabular}

Table 4: $\xi\left(n_{\text {opt }}\right), n_{\text {opt }}, R_{1}\left(n_{\text {opt }}\right), R_{2}\left(n_{\text {opt }}\right)$ with respect to $\theta_{2}$ (and $\varepsilon$ ), Example 3, case $\sigma=0.5$ (and $\left.n_{\max }=50\right)$

\begin{tabular}{ccccrr}
\hline$\theta_{2}$ & $\varepsilon$ & $\xi\left(n_{\text {opt }}\right)$ & $n_{\text {opt }}$ & $R_{1}\left(n_{\text {opt }}\right)$ & $R_{2}\left(n_{\text {opt }}\right)$ \\
\hline 1.25 & 0.005 & 86.75 & 76 & $-6.94 \times 10^{-17}$ & $-4 \times 10^{-3}$ \\
1.50 & 0.005 & 24.38 & 19 & $-4.16 \times 10^{-17}$ & $-4 \times 10^{-3}$ \\
1.75 & 0.025 & 11.49 & 8 & $-4.16 \times 10^{-17}$ & $-1.8 \times 10^{-2}$ \\
2 & 0.025 & 7.76 & 5 & $-4.16 \times 10^{-17}$ & $9.3 \times 10^{-3}$ \\
2.25 & 0.025 & 5.14 & 3 & $-4.16 \times 10^{-17}$ & $-7.1 \times 10^{-3}$ \\
2.50 & 0.025 & 3.75 & 2 & $-4.16 \times 10^{-17}$ & $-1.8 \times 10^{-2}$ \\
2.75 & 0.075 & 3.74 & 2 & $-4.16 \times 10^{-17}$ & $5.1 \times 10^{-2}$ \\
3 & 0.075 & 2.23 & 1 & $-4.16 \times 10^{-17}$ & $-5.3 \times 10^{-2}$ \\
4 & 0.1 & 2.23 & 1 & $-4.16 \times 10^{-17}$ & $9.1 \times 10^{-2}$ \\
\hline
\end{tabular}

Table 5: $\xi\left(n_{\text {opt }}\right), n_{\text {opt }}, R_{1}\left(n_{\text {opt }}\right), R_{2}\left(n_{\text {opt }}\right)$ with respect to $\theta_{2}$ (and $\varepsilon$ ), Example 3, case $\sigma=0.75$ (and $\left.n_{\max }=100\right)$

\begin{tabular}{ccccrr}
\hline$\theta_{2}$ & $\varepsilon$ & $\xi\left(n_{\text {opt }}\right)$ & $n_{\text {opt }}$ & $R_{1}\left(n_{\text {opt }}\right)$ & $R_{2}\left(n_{\text {opt }}\right)$ \\
\hline 1.25 & 0.025 & 9.16 & 8 & $-4.16 \times 10^{-17}$ & $-1.8 \times 10^{-2}$ \\
1.35 & 0.025 & 4.82 & 4 & $6.94 \times 10^{-17}$ & $-2.4 \times 10^{-2}$ \\
1.45 & 0.050 & 3.71 & 3 & $-4.16 \times 10^{-17}$ & $3.0 \times 10^{-2}$ \\
1.75 & 0.050 & 1.41 & 1 & $-4.16 \times 10^{-17}$ & $1.2 \times 10^{-2}$ \\
2 & 0.1 & 1.41 & 1 & $-4.16 \times 10^{-17}$ & $9.1 \times 10^{-2}$ \\
2.25 & 0.1 & 1.41 & 1 & $-4.16 \times 10^{-17}$ & $1.0 \times 10^{-1}$ \\
2.50 & 0.1 & 1.41 & 1 & $-4.16 \times 10^{-17}$ & $1.0 \times 10^{-1}$ \\
3 & 0.1 & 1.41 & 1 & $-4.16 \times 10^{-17}$ & $1.0 \times 10^{-1}$ \\
\hline
\end{tabular}

Table 6: $\xi\left(n_{\text {opt }}\right), n_{\text {opt }}, R_{1}\left(n_{\text {opt }}\right), R_{2}\left(n_{\text {opt }}\right)$ with respect to $\theta_{2}$ (and $\varepsilon$ ), Example 3, case $\sigma=0.25\left(\right.$ and $\left.n_{\max }=10\right)$ 


\subsection{Comparison of Sampling Plans I and II}

In Sections 2 and 3, we have proposed two types of sampling plans. Sampling Plan I is based on the statistic $\sum_{i=1}^{n} I\left(W_{i}>\xi\right)$, whereas Sampling Plan II is based on $\sum_{i=1}^{n} W_{i}$. The optimal parameters for each sampling plan are given in Tables 1-3 and Tables 4-6, respectively. As there are negligible differences in $R_{1}$ and $R_{2}$, we compare the total number of items used in the testing procedure. Especially when there is very small difference between $\theta_{1}$ and $\theta_{2}$, the total number of items used in the testing procedure $n$ of Sampling Plan II is much smaller than that of Sampling Plan I. Thus, from the experimental comparison, we can conclude that Sampling Plan II spends less items in the degradation test. Furthermore, Sampling Plan II has smaller number of parameters and Algorithm 2 is much simpler than Algorithm 1. In addition, in Sampling Plan II, the parameter $\xi$ can be conveniently obtained by using the Cdf of the normal distribution.

On the other hand, in some cases, whether the amount of degradation of an item reaches a predetermined level $\xi$ (i.e., $W_{i}>\xi$ ) or not can be easily checked and directly reported by a censor built in the item. In this case, in applying Sampling Plan I, there is no need to observe the degradation levels of the items on the test and the observation cost can be substantially saved. In this case, Sampling Plan I would be preferable from an "economical point of view".

\section{Concluding Remarks}

In this paper, based on the observation of the deterioration of the items, two types of variables acceptance sampling plans have been developed. The first type of sampling plan (Plan I) is based on the statistic $\sum_{i=1}^{n} I\left(W_{i}>\xi\right)$ and the second type (Plan II) is based on $\sum_{i=1}^{n} W_{i}$. Considering both the producer's risk and consumer's risk, efficient algorithms to determine the parameters of the sampling plans have been suggested. Furthermore, the effect of the proposed sampling plans on the accepted lots has been analyzed. It has been shown that the reliability characteristic of the accepted items after the test improves compared with that of the items before the test in a stochastic sense. Finally, we have compared the two types of sampling plans from both the economical and technical perspectives.

In this paper, we have considered the Wiener process with positive drift as the degradation process of the items. If more appropriate, other types of degradation processes could be employed and similar sampling plans could be developed based on them.

\section{Acknowledgments}

The authors thank the reviewer for helpful comments and advices. The work of the first author was supported by the National Research Foundation of Korea (NRF) grant funded by the Korea government (MSIP) (No. 2019R1A2B5B02069500). The work of the first author was also supported by Basic Science Research Program through the National Research Foundation of Korea (NRF) funded by the Ministry of Education (Grant Number: 2019R1A6A1A11051177).

\section{References}


Aslam M, Jun CH (2009) A group acceptance sampling plan for truncated life test having Weibull distribution. Journal of Applied Statistics 36:1021-1027.

Aslam M, Kundu D, Ahmad M (2010) Time truncated acceptance sampling plans for generalized exponential distribution. Journal of Applied Statistics 37:555-566.

Aslam M, Jun CH, Ahmad M (2011a) New acceptance sampling plans based on life tests for Birnbaum-Saunders distributions. Journal of Statistical Computation and Simulation 81:461-470.

Aslam M, Jun CH, Ahmad M (2011b) A two-stage group sampling plan based on truncated life tests for a general distribution. Journal of Statistical Computation and Simulation 81:1927-1938.

Aslam M, Balamurali S, Jun CH, Rasool M (2013a) Optimal designing of skip-lot sampling plan of type SkSP-2 with group acceptance sampling plan as reference plan under Burr-type XII distribution. Journal of Statistical Computation and Simulation 83:37-51.

Aslam M, Yen CH, Chang CH, Jun CH (2013b) Multiple states repetitive group sampling plans with process loss consideration. Applied Mathematical Modelling 7:9063-9075.

Aslam M, Azam M, Jun CH (2013c) A mixed repetitive sampling plan based on process capability index. Applied Mathematical Modelling 37:10027-10035.

Balakrishnan N, Balasubramanian K, Viveros R (1993) On sampling inspection plans based on the theory of runs. The Mathematical Scientist 18:113-126.

Balakrishnan N, Leiva V, Lopez J (2007) Acceptance sampling plans from truncated life tests based on the generalized Birnbaum-Saunders distribution. Communications in Statistics - Simulation and Computation 36:643-656.

Blugren W, Hewette J (1973). Double sampling test for hypotheses about the mean of an exponential distribution. Technometrics 22:421-426.

Cha JH (2016) Analysis of reliability characteristics in the acceptance sampling tests. Journal of Applied Statistics 43:1874-1891.

Cha JH (2015) Variables acceptance reliability sampling plan for repairable items. Statistics 49:1141-1156.

Cha JH, Badia FG (2020a) Variables acceptance reliability sampling plan based on degradation test. Statistical Papers https://doi.org/10.1007/s00362-020-01185-1.

Cha JH, Badia FG (2020b) Variables acceptance reliability sampling plan for items subject to inverse Gaussian degradation process. Journal of Applied Statistics https://doi.org/10.1080/02664763.2020.1723505. 
Chen J, Choy STB, Li KH (2004) Optimal Bayesian sampling acceptance plan with random censoring. European Journal of Operational Research 155:683-694.

Doksum KA, Hoyland A (1992) Models for variable-stress accelerated life testing experiments based on Wiener processes and the inverse Gaussian distribution. Technometrics 34:74-82.

Epstein B (1954) Truncated life tests in exponential case. Annals of Mathematical Statistics 25:555-564.

Epstein B, Sobel M (1953) Life testing. Journal of American Statistical Association 48:485-502.

Epstein B, Sobel M (1955) Sequential life test in the exponential case. Annals of Mathematical Statistics 26:82-93.

Fairbanks K (1988) A two-stage life test for the exponential parameter. Technometrics 30:175-180.

Fernández AJ, Pérez-Gonzalez CJ, Aslam M, Jun CH (2011) Design of progressively censored group sampling plans for Weibull distributions: An optimization problem. European Journal of Operational Research 211:225-532.

Fertig KW, Mann NR (1980) Life test sampling plans for two-parameter Weibull population. Technometrics 22:165-177.

Folks JL, Chhikara RS (1978) The inverse Gaussian distribution and its statistical application-A review. Journal of the Royal Statistical Society, Series B. 40:263289.

Hu CH, Lee MY, Tang J (2015) Optimum step-stress accelerated degradation test for wiener degradation process under constraints. European Journal of Operational Research 241:412-421.

Jin G, Matthews D (2014) Reliability demonstration for long-life products based on degradation testing and a Wiener process model. IEEE Transactions on Reliability 63:781-797.

Kahle W, Mercier S, Paroissin C (2016) Degradation processes in reliability. John Wiley \& Sons, Inc., Hoboken, NJ.

Klenke A, Mattner L (2010) Stochastic ordering of classical discrete distributions. Advances in Applied Probability 42:392-410.

Lee H, Cha JH (2017) Reliability sampling plan for repairable items following general failure process and its statistical analysis. Statistics 50:1159-1178.

Lu CJ, Meeker MQ (1993) Using degradation measures to estimate a time-to-failure distribution. Technometrics 35:161-171. 
Montgomery DC (2012) Introduction to statistical quality control, 7th edition. Wiley, New Jersey.

Müller A, Stoyan D (2002) Comparison methods for stochastic models and risks. Wiley, Chichester.

Pan D, Liu JB, Cao J (2016) Remaining useful life estimation using an inverse Gaussian degradation model. Neurocomputing 185: 64-72.

Pan D, Liu JB, Huang F, Cao J, Alsaedi A (2017) A Wiener process model with truncated normal distribution for reliability analysis. Applied Mathematical Modelling 50: 333-346.

Schneider H (1989) Failure-censored variables sampling plans for lognormal and Weibull distributions. Technometrics 31:199-206.

Shaked M, Shanthikumar JG (2007) Stochastic orders. Springer, New York.

Si XS, Wang W, Hu CH, Chen MY, Zhou DHA (2013) Wiener-process-based degradation model with a recursive filter algorithm for remaining useful life estimation. Mechanical Systems and Signal Processing 35:219-237.

Stephens KS. The handbook of applied acceptance sampling: Plans, procedures and principles. ASQ Quality Press, Milwaukee.

Tsai TR, Wanbo L (2009) Interval censored sampling plans for the gamma lifetime model. European Journal of Operational Research 192:116-124.

Tsai CC, Lin CT, Balakrishnan N (2015) Optimal design for accelerated-stress acceptance test based on Wiener process. IEEE Transactions on Reliability 64:603-612.

Van Noortwijk J (2009) A survey of the application of Gamma processes in maintenance. Reliability Engineering \& System Safety 94:2-21.

Wang X (2010) Wiener processes with random effects for degradation data. Journal of Multivariate Analysis 101:340-351.

Wang X, Xu D (2010) An inverse Gaussian process model for degradation data. Technometrics 52:188-197.

Whitmore GA (1995) Lifetime data analysis. pp. 1-307, 1995.

Wu CW, Shu MH, Chang YN (2018) Variable-sampling plans based on lifetime-performance index under exponential distribution with censoring and its extensions. Applied Mathematical Modelling 55:81-93.

Zhang Z, Si X, Hu C, Lei Y (2018) Degradation data analysis and remaining useful life estimation: A review on Wiener-process-based methods. European Journal of Operational Research 271:775-796. 